\title{
Stop neglecting fungi
}

\section{Fungal pathogens are virtually ignored by the press, the public and funding bodies, despite posing a significant threat to public health, food biosecurity and biodiversity.}

Fungal infections will probably not have made major news today, perhaps not even this week or month. Indeed, in comparison to the threat from drug-resistant bacterial infections or viral outbreaks, diseases caused by fungi, fungal drug resistance and the development of new antifungal therapeutics gets little coverage. Yet in this case, no news is certainly not good news, and the disparity relative to other infectious disease agents unjustified. The word fungus usually evokes images of athlete's foot, unseemly looking nails, or scrumptious cheese and mouth-watering mushrooms. However, few realize that over 300 million people suffer from serious fungal-related diseases, or that fungi collectively kill over 1.6 million people annually ${ }^{1}$, which is more than malaria and similar to the tuberculosis death toll. Fungi and oomycetes destroy a third of all food crops each year, which would be sufficient to feed 600 million people. Furthermore, fungal infestation of amphibians has led to the largest disease-caused loss of biodiversity ever recorded, while fungi also cause mass mortality of bats, bees and other animals, and decimate fruit orchards, pine, elm and chestnut forests ${ }^{2}$. Headline-grabbing statistics, one would imagine.

There are an estimated 1.5 million fungal species $^{3}$, of which over 8,000 are known to cause disease in plants and 300 to be pathogenic to humans. Candida, Aspergillus, Pneumocystis and Cryptococcus spp. are the most common cause of serious disease in humans, and five diseases - wheat stem rust, rice blast, corn smut, soybean rust and potato late blight - are the most devastating for crop production. Infections primarily occur in immunocompromised patients, such as those undergoing chemotherapy or infected with HIV, and many are acquired in hospitals. However, infections of otherwise healthy people are on the rise. Global warming is inducing rapid poleward movement of crop fungal pathogens, and may also increase the prevalence of fungal disease in humans as fungi adapt to survival in warmer temperatures ${ }^{4}$. In this scenario, increasing resistance to the limited arsenal of antifungal drugs is a serious concern ${ }^{5}$, especially for Candida and Aspergillus infections, for which the therapeutic options have become limited. The emergence of multi-drug resistant Candida glabrata and
Candida auris is a global health threat ${ }^{6}$, and azole-resistant Aspergillus has up to $30 \%$ prevalence in some European hospitals, which report higher than $90 \%$ mortality rates ${ }^{7}$.

Experts agree that fungal pathogens are a serious threat to human health, food biosecurity and ecosystem resilience, yet lack of funding translates into inadequate surveillance systems to monitor fungal disease incidence and antifungal drug resistance, which often rely on not-for-profit initiatives, such as the Global Action Fund for Fungal Infections (GAFFI; http://www. gaffi.org/). As highlighted in the World Health Organization (WHO) Global Report on Antimicrobial Resistance Surveillance ${ }^{8}$, which devotes fewer than $10 \%$ of its pages to fungi, resources allocated for monitoring and reducing antifungal drug resistance are limited. Indeed, the WHO has no funded programmes specifically targeting fungal diseases, fewer than 10 countries have national surveillance programs for fungal infections, and fewer than 20 have fungal reference diagnostic laboratories. Many of the diagnostic tests that do exist are not available in developing countries, and well-established antifungal drugs such as amphotericin B, flucytosine and cotrim - that would cure disease do not reach people that need them, a large fraction of which are in sub-Saharan Africa ${ }^{9}$. In an attempt to tackle this silent humanitarian crisis, organizations such as GAFFI, the US Centres for Disease Control, Médecins Sans Frontières and Clinton Health Access lobbied to include amphotericin B and flucytosine on the WHO Essential Medicines List ${ }^{9}$. Beyond this, GAFFI has put forth a roadmap to achieve diagnosis and access to antifungals for $95 \%$ of infected people by 2025 , which aims to improve the availability/affordability of diagnostics, train clinicians in fungal disease diagnosis and treatment, and ensure that antifungals are available globally ${ }^{10}$. Funding is also urgently needed to advance our understanding of fungal pathogenesis and drug resistance, develop new diagnostics and antifungal strategies, and improve monitoring of infection and antifungal resistance, as this will ultimately inform new strategies to tackle fungal infections.

Why then do fungi remain stubbornly off the mainstream radar? A possible reason is that most people think of fungi as causing infections that are uncomfortable but relatively easy to address, as invasive, life-threatening disease impacts few people in developed countries. In addition, our human-centric view of the world limits the amount of attention devoted to plant health, even if this directly impacts food availability. Bacteria and viruses have historically received more attention, in part because of the simple (yet not always correct) narrative to portray them as harmful, whereas fungi and their products can be edible, or useful drugs, and they are used as model organisms for understanding higher eukaryotes. Nevertheless, bringing emerging fungal threats into better focus for the broader research community, funders, media organizations and the general public should be a priority and will catalyse support and progress for this important and neglected group of pathogens.

Something that should make headlines this month, but may not, is the opening in South Africa of the AFGrica Unit in Medical Mycology, the first international research centre for tackling fungal infections (http:// go.nature.com $/ 2 \mathrm{sN} 8 \mathrm{x} 0 \mathrm{z}$ ). This centre is an initiative of the University of Aberdeen Fungal Group (now the MRC Centre for Medical Mycology), in conjunction with the University of Cape Town. It will benefit from a Wellcome Trust Strategic Award that funds $\mathrm{PhD}$ students from developing nations to train in Aberdeen and other medical mycology centres and return home to help address fungal research and training needs. International collaborative efforts such as this one will be essential to give fungal diseases the prominence they require, and as such they should be encouraged. It is time to stop the neglect and put fungal diseases firmly in the spotlight.

\footnotetext{
References

1. The Burden of Fungal Disease (LIFE, 2017); http://go.nature.com/2sMKpuN

Fisher, M. C. et al. Nature 484, 186-194 (2012).

3. Hawksworth, D. L. Mycol. Res. 105, 1422-1432 (2001).

4. Garcia-Solache, M. A. \& Casadevall, A. mBio 1, e00061-10 (2010).

5. Fairlamb, A. H., Gow, N. A., Matthews, K. R. \& Waters, A. P. Nat. Microbiol. 1, 16092 (2016)

6. Candida auris (CDC, 2017); http://go.nature.com/2svZBZb

7. van Paassen, J. et al. Eurosurveill. 21, 3 (2016).

8. Antimicrobial Resistance: Global Report on Surveillance (WHO, 2014); http://go.nature.com/2tTi59N

9. Hidden Crisis (GAFFI, 2016); http://go.nature.com/2tPghPf 10. GAFFI Roadmap (GAFFI, 2015); http://go.nature.com/2swH1Ae
} 


\section{Correction: Stop neglecting fungi}

Nature Microbiology 2, 17120 (2017); published 25 July 2017; corrected 31 July 2017.

The original version of this Editorial referred to a set of five diseases (wheat stem rust, rice blast, corn smut, soybean fungi and potato late blight) as fungal diseases, which was incorrect as potato late blight is caused by an oomycete. In addition, 'soybean fungi' has been replaced with 'soybean rust'. We apologize for any confusion that may have been caused; all versions of the Editorial have now been modified to rectify these errors. 